\title{
A New Catheter Technique to Correct Severe IVC Filter Tilt during Placement
}

\author{
Sandeep T. Laroia ${ }^{1}$ Justin J. Guan ${ }^{1, \odot}$ Archana T. Laroia ${ }^{1} \quad$ Lucas Lenhart $^{1}$ Antony J. Hayes ${ }^{1}$ \\ ${ }^{1}$ Division of Interventional Radiology, Department of Radiology, \\ University of lowa Hospitals and Clinics, lowa City, lowa, \\ United States \\ Address for correspondence Justin J. Guan, MD, Department of \\ Radiology, University of lowa Hospitals and Clinics, 200 Hawkins Drive, \\ lowa City, IA 52242, United States (e-mail: Justin-guan@uiowa.edu).
}

\begin{abstract}
Introduction Inferior vena cava (IVC) filter tilt is a common complication that occurs during and after filter placement. Severe tilting leads to reduced filter efficacy, lower retrieval success, and higher complication rates during retrieval. We present a novel catheter technique to correct severely tilted cone-shaped IVC filters without having to retrieve and replace the existing filter.

Methods A retrospective review was performed for patients at our institution over three years who had severely tilted filters and underwent correction with the catheter technique. Indications for filter placement were categorized, and patient age, gender, tilt correction outcome, and complication rates were collected and analyzed. After severe tilting was noted on post-IVC filter deployment venogram, a Sos catheter was passed via the same femoral access site used for the filter placement. The catheter tip was reformed inside the cone of the filter and was used to push the filter tip back toward midline. Completion venogram was taken to document the amelioration of the tilt.

Results Out of 28 patients who were found to have severely tilted filters on deployment and underwent correction with the catheter technique, $27 / 28$ (96.4\%) had successful correction. One (3.6\%) had a minor complication where the filter struts became entangled with the catheter tip; however, simple maneuvering of the catheter and use

Keywords

- inferior vena cava

- filter

- tilt of a stiff wire to straighten the catheter loop freed up the entanglement. No major complications occurred.

Conclusion This technique is safe, effective, obviates filter replacement, and can be considered an additional management option for severe IVC filter tilt during placement.
\end{abstract}

\section{Introduction}

A common technical difficulty associated with inferior vena cava (IVC) filter (IVCF) placement is angulation or tilt of the filter, occurring in up to $5.5 \%$ of IVCF procedures..$^{1-5}$ At more than 15 degrees, filter tilting may lead to reduced efficacy of the filter in preventing pulmonary embolism (PE) and reduced success rate of retrieving the filter once it is no longer indicated..$^{2,3,6}$ Severe tilting leads to compromise of the conical design, and the larger resulting openings for passage of clot increases the risk of significant PE. Deviation of the filter hook away from the IVC midline can also cause difficulties in removing the filter using standard techniques, especially if the hook is against the caval wall. ${ }^{2}$ Severe filter tilting is also a leading cause of increased complication rates associated with advanced retrieval techniques, especially if the filter tip is subsequently embedded deep within the caval wall. $4,7,8$ Major complications include IVC dissection, IVC thrombus/stenosis, filter fracture with embedded strut, and IVC injury with hemorrhage. ${ }^{1,4,7,8}$
DOI https://doi.org/

10.1055/s-0039-3401346

ISSN 2457-0214.
License terms

$($ () (1) $\Theta \circledast$ 
Mitigating severe IVCF tilting has traditionally been filter retrieval and replacement, but the process of having to retrieve and replace the existing filter is itself associated with procedural risks and longer radiation exposure. Moreover, retrieval of a tilted filter has been found to prolong procedure time even further. ${ }^{2}$ Another logical option would be to correct the filter tilt on deployment as soon as severe tilting is found; however, the existing literature on correction techniques is limited. Knott et al in 2012 presented an Amplatz wire technique to correct severely tilted IVCFs. This technique was used only on the Cook Celect (Cook Medical) IVC filter, and only a limited number of cases were reported. ${ }^{2}$

The purpose of this study is to present a new catheter technique to correct severely tilted conical IVCFs during placement using a Sos catheter, without having to retrieve and replace the existing filter.

\section{Methods}

After institutional review board approval, retrospective review was performed for all patients at a single institution found to have severely tilted conical IVCFs on deployment and underwent tilt correction using the catheter technique described next between 2013 and 2015. The indications for filter placement were categorized based on the 2006 Society of Interventional Radiology (SIR) Consensus Guidelines. ${ }^{9}$ Severe tilt was defined as deviation by more than 15 degrees of the filter long axis with respect to the long axis of the vena cava ( - Fig. 1a). Tilt correction success was defined as correction of filter tilt to less than 5 degrees with respect to vena cava midline as documented by postprocedural venogram. All measurements were performed point-of-care using the inbuilt software of the Siemens Axiom Artis Angiography System (Siemens Medical Solutions, Inc.) with standard, single-plane anteroposterior venography. Patient age, gender, tilt correction outcome, and complication rates were collected and analyzed. Minor and major procedural complications were defined according to the SIR clinical practice guidelines. ${ }^{10}$ The correction technique was performed by a single interventional radiologist.

Catheter correction was performed for any case of severe filter tilting noted on post IVC filter deployment venogram (-Figs. 1, 2a). Using the same femoral access through which the filter was placed, a Bentson guidewire (Cook Medical) was inserted and advanced across the filter. Once the wire was adequately advanced, a Sos catheter (AngioDynamics) was placed over the wire and advanced to just below the filter. The wire was carefully pulled back into the shaft of the catheter so that the catheter tip could reform ( - Fig. $\mathbf{2 b}$ ). The catheter shaft was curved against the caval wall on the side of the tilt for counter pressure, and the catheter tip was then used to push the tip of the cone carefully toward the center of the IVC ( - Fig. 2c). Once adequate correction was achieved, the catheter was very carefully pulled back to avoid entanglement in the filter struts. Once below the level of the filter, the Bentson guidewire was readvanced into the catheter to straighten the curved tip. The catheter was removed and a completion venogram was taken to document the amelioration of the tilt ( - Fig. 2d).
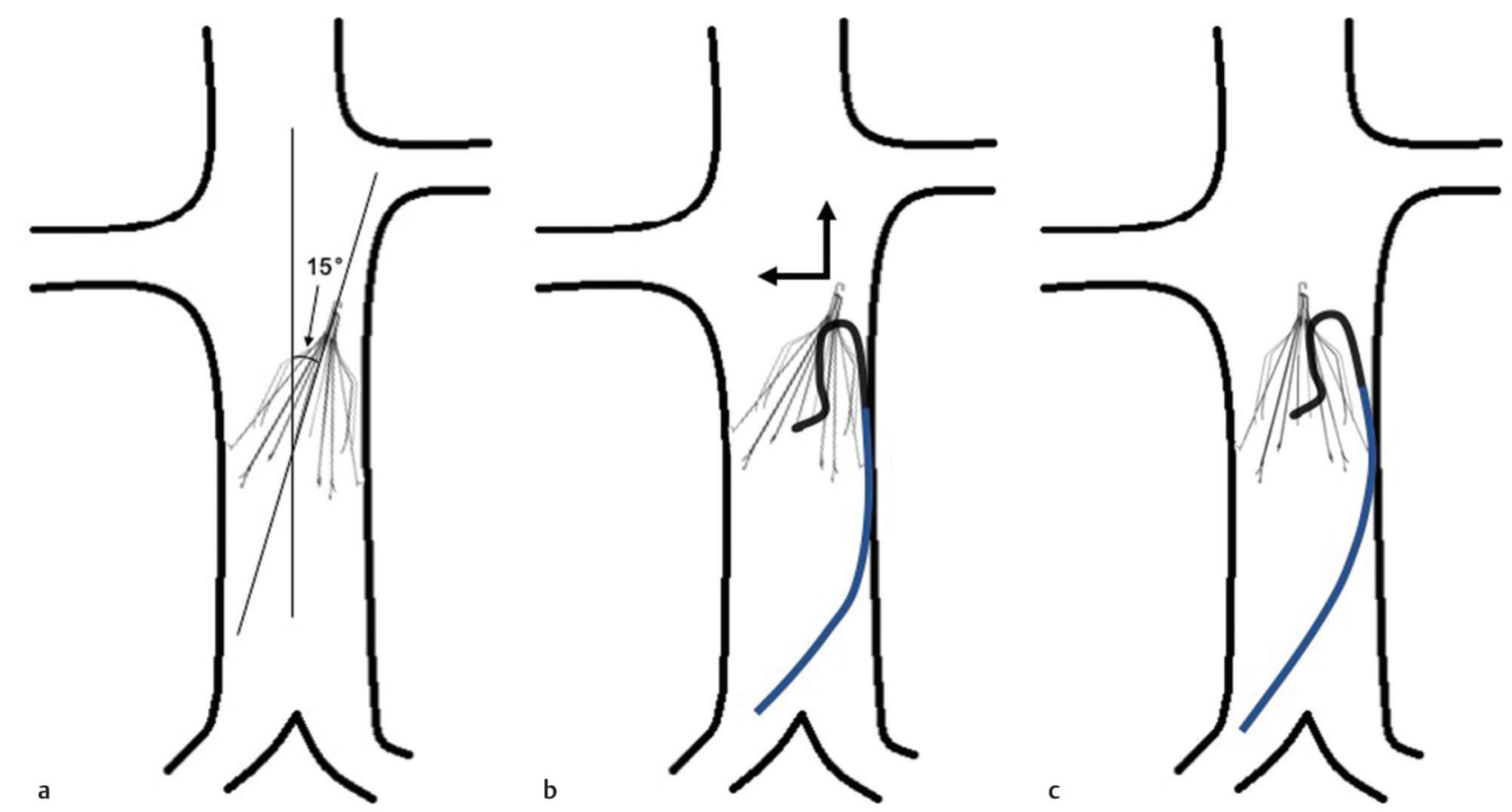

Fig. 1 Diagrams depicting filter tilt measurement and the Sos catheter technique. (a) The tilt angle is measured between the long axis of the vena cava and the long axis of the IVC filter. (b) The Sos catheter is reformed just underneath the filter, and the catheter tip is used to push the IVC filter tip toward midline. (c) The catheter shaft is curved against the vena cava wall for counter pressure while pushing the IVC filter tip back into alignment. 

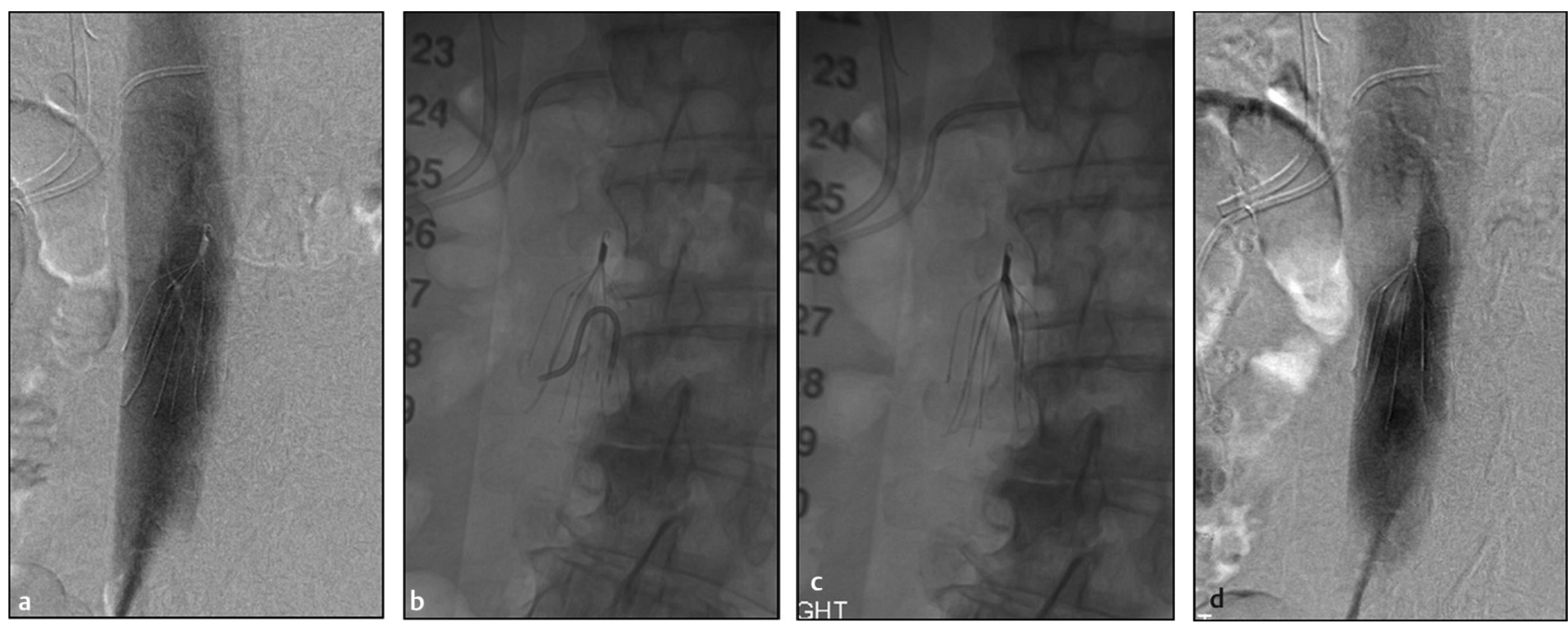

Fig. 2 Correction of severe IVC filter tilt in a 75-year-old male using the Sos catheter technique. (a) Post IVC filter deployment venogram shows a severely tilted IVC filter, which measures $16.4^{\circ}$ with respect to the vena cava midline. (b) A Sos catheter is then advanced over a Bentson guidewire and its tip reformed just underneath the IVC filter cone. (c) Catheter shaft is curved against the IVC wall, and catheter tip is used to push the filter tip toward the vena cava midline. (d) Catheter is removed and completion venogram taken to document amelioration of tilt.

Table 1 Demographics of patients undergoing Sos catheter correction for severely tilted IVC filters

\begin{tabular}{|l|l|}
\hline Mean age (range) & 56.1 years (16-94) \\
\hline Male:female ratio (\%) & $17: 11(61: 39)$ \\
\hline Types of filter placed & \\
\hline Argon Option & $7(25 \%)$ \\
\hline Bard Eclipse & $6(21 \%)$ \\
\hline Bard Denali & $15(54 \%)$ \\
\hline Indications for filter placement & \\
\hline Prophylactic & $19(68 \%)$ \\
\hline Post trauma & $8(29 \%)$ \\
\hline Preoperative & $11(29 \%)$ \\
\hline $\begin{array}{l}\text { Absolute (previous anticoagulation } \\
\text { complicated by gastrointestinal bleed) }\end{array}$ & $9(32 \%)$ \\
\hline Complications & $1(3.6 \%)$ \\
\hline Minor & $0(0 \%)$ \\
\hline Major &
\end{tabular}

\section{Results}

Out of 170 IVCFs placed between 2013 and 2015, 28 IVCFs in 28 patients were found to have severe tilt on deployment (-Table 1). Seventeen patients were male and 11 patients were female. The mean age was 56.1 years (range, 16-94 years). Indications for filter placement were prophylactic in 19 patients (posttrauma in 8 and preoperative in 11) and absolute in 9 with previous medical anticoagulation complicated by GI bleeding (-Table 1). The filter types included Option (Argon) in 7 patients, Eclipse (Bard) in 6, and Denali (Bard) in 15. All corrections were performed immediately after severe tilt was diagnosed on postdeployment venogram.

Out of 28 patients, 27 (96.4\%) had successful adjustment of their filters back to midline of the IVC. No major complication occurred. There was one (3.6\%) minor complication where the filter struts became entangled with the catheter tip; however, this responded to simple maneuvering of the catheter and use of a stiffer wire to straighten the loop of the catheter. Twenty-three filters were subsequently removed at time periods ranging from 3 weeks to 9 months, once no longer indicated. There were no complications associated with their retrievals. Of the five filters left in, three were due to the patients' ongoing clinical conditions while two were due to the patients being lost to follow-up.

\section{Discussion}

Severe filter tilting leads to both reduced filter efficacy as well as reduced retrieval success once the filter is no longer indicated. In fact, many studies have found that the factor most associated with failed IVCF retrieval using standard techniques is filter tilt. ${ }^{6-8}$ Although advanced retrieval techniques have higher success rates of up to $95 \%$, severe tilt is also a leading factor associated with major complications during advanced techniques, including IVC dissection, IVC thrombus/stenosis, filter fracture with embedded strut, IVC injury with hemorrhage, and vascular injury from complicated venous access. Severe filter tilt can be managed with retrieval and replacement, but the process of having to retrieve and replace the existing filter is itself associated with procedural risks and longer radiation exposure.

An alternative option is correction during placement, but to the authors' knowledge, the only published correction technique to date is the Amplatz wire technique presented by Knott et al in 2012. This technique involves advancing an Amplatz wire through a Y-connector side port on the filter sheath to "straighten" any IVCFs that appeared severely tilted while still partially unsheathed, successfully preventing 11 filter tilts that were studied. The major limitations of 
this study were that (1) the only filter type investigated was the Celect filter, which is only one of several different types of conical retrievable filters available, potentially limiting the application of this technique to the correction of other retrievable filters, and (2) as the authors stated in the study, the tilt measurements were performed before filters were fully deployed, which may overestimate the tilt angles.

In this study, we present a novel technique of using a Sos catheter to correct severely tilted IVCFs placed from a femoral approach, successfully correcting $27 / 28$ (96.4\%) filters. There were no major complications, and similar to the theoretical risk in the Amplatz wire technique of entangling the Amplatz wire to the filter struts, we experienced one case of entanglement between the catheter tip and the filter struts. This was easily corrected with maneuvering of the catheter and using a stiffer wire to straighten the catheter loop. Our technique was performed on three different types of conical filters with high success, increasing the applicability of this technique to all conical filters. Filter tilt was measured on postplacement venogram after full deployment of the IVCFs, increasing our confidence that all 28 filters met the inclusion criteria of being severely tilted.

It is important to note that the Sos catheter technique is meant to be performed the same day as filter placement. We observed anecdotally that if the tilt is not corrected within first few minutes of noticing the tilt, that is, waiting for arrival of the Sos catheter from the supply room, the subsequent correction becomes much harder, although still achievable. This is possibly due to embedding of filter struts within the wall of the vena cava even this early after placement. We recommend that this technique be utilized as quickly as possible after discovering the tilt. In our practice, we keep a Sos catheter ready on standby for all filter placements by the femoral route. When performing the correction, care must be taken to make sure the catheter tip does not cross the cone of the filter, as this will increase the risk of catheter-strut entanglement. It is also important to make sure that the catheter shaft is curved against the IVC wall on the side of the tilt for counter pressure, as otherwise the tilt may worsen.

\section{Conclusion}

The Sos catheter technique to correct severely tilted IVCFs has a high success rate of $96.4 \%$. This technique is safe and effective, obviates the need for filter retrieval and replacement, and can be considered an additional management option for severe IVCF tilt during placement from a femoral approach. Further studies are warranted looking at whether correcting severe filter tilt upon placement truly leads to improved IVCF efficacy, improved filter retrieval success, and/or reduced retrieval complications.

\section{Conflicts of Interest}

None.

\section{Acknowledgments}

None.

\section{References}

1 Al-Hakim R, Kee ST, Olinger K, Lee EW, Moriarty JM, McWilliams JP. Inferior vena cava filter retrieval: effectiveness and complications of routine and advanced techniques. J Vasc Interv Radiol 2014;25(6):933-939

2 Knott EM, Beacham B, Fry WR. New technique to prevent tilt during inferior vena cava filter placement. J Vasc Surg 2012;55(3):869-871

3 Al-Hakim R, McWilliams JP, Derry W. Kee ST. The hangman technique: a modified loop snare technique for the retrieval of inferior vena cava filters with embedded hooks. J Vasc Interv Radiol 2015;26(1):107-110

4 Iliescu B, Haskal ZJ. Advanced techniques for removal of retrievable inferior vena cava filters. Cardiovasc Intervent Radiol 2012;35(4):741-750

5 Bos AS, Tullius T, Patel M, et al. Indwelling and retrieval complications of Denali and Celect infrarenal vena cava filters. J Vasc Interv Radiol 2016;27(7):1021-1026

6 Renno A, Khateeb F, Kazan V, et al. A single center experience with retrievable IVC filters. Vascular 2015;23(4):350-357

7 Kuo WT, Odegaard JI, Louie JD, et al. Photothermal ablation with the excimer laser sheath technique for embedded inferior vena cava filter removal: initial results from a prospective study. J Vasc Interv Radiol 2011;22(6):813-823

8 Stavropoulos SW, Dixon RG, Burke CT, et al. Embedded inferior vena cava filter removal: use of endobronchial forceps. J Vasc Interv Radiol 2008;19(9):1297-1301

9 Kaufman JA, Kinney TB, Streiff MB, et al. Guidelines for the use of retrievable and convertible vena cava filters: report from the Society of Interventional Radiology multidisciplinary consensus conference. J Vasc Interv Radiol 2006;17(3):449-459

10 Sacks D, McClenny TE, Cardella JF, Lewis CA. Society of Interventional Radiology clinical practice guidelines. J Vasc Interv Radiol 2003;14(9 Pt 2):S199-S202 